\title{
LETTER
}

\section{Severe hepatitis associated with sitaxentan and response to glucocorticoid therapy}

\section{To the Editors:}

Endothelin receptor antagonists (ERA) have become standard therapy for patients with pulmonary arterial hypertension (PAH), next to phosphodiesterase-5 inhibitors and prostanoids [1-3]. One of the most common side-effects associated with ERA therapy is hepatotoxicity, usually detected by an increase in the serum aminotransferases $[1,4]$. The underlying mechanism may be related to inhibition of a bile-salt transporter pump [5]. The typical pattern of this type of liver injury is that of a toxic mechanism, i.e. dose-related and rapidly reversible upon dose reduction or drug withdrawal.

Herein, we present a case of liver injury associated with the ERA sitaxentan, which showed a different pattern in that liver injury worsened despite drug discontinuation and aminotransferases normalised after glucocorticoid therapy had been initiated, raising the hypothesis that hepatotoxicity might have been related to an idiosyncratic, i.e. immune-mediated, mechanism.

The patient, a 25-yr-old female (height: $178 \mathrm{~cm}$; weight: $58 \mathrm{~kg}$ ) with Eisenmenger's syndrome, due to an uncorrected ventricle septum defect, was referred to our institution (Hannover Medical School, Hannover, Germany) in March 2007 when she presented in functional class III with a 6 min walking distance (6MWD) of $372 \mathrm{~m}$. Her past medical history was otherwise unremarkable. She had no history of liver disease or autoimmune disorders. Her medication consisted of digoxin and acetyl salicylic acid, both started $>10$ yrs previously.

PAH-targeted therapy was initiated with bosentan in March 2007. The patient had functional improvement and the 6MWD increased to $413 \mathrm{~m}$ after 4 months of therapy. As shown in the figure 1, liver aminotransferase levels were normal during the first 6 months of bosentan therapy but then increased (alanine aminotransferase (ALT) to $\sim 4 \times$ upper level of normal and aspartate aminotransferase (AST) to $\sim 2 \times$ upper level of normal). A follow-up test 2 weeks later showed a further increase in these liver enzymes. At that time, bosentan was stopped and the aminotransferase levels normalised within 2 weeks.

Since the exercise capacity of this patient deteriorated slightly after withdrawal of bosentan it was decided to start sitaxentan therapy $\sim 3$ months after discontinuation of bosentan. At that time, hepatic aminotransferase levels were in the normal range and remained so for the first 3 months of therapy. However, after 4 months, laboratory tests showed a marked increase in the AST and ALT levels (fig. 1), upon which sitaxentan was stopped immediately. The patient denied the intake of any other drugs or alcohol. An abdominal ultrasound showed a normal liver structure and no signs of hepatic venous congestion. Clinical examination and echocardiography showed no signs of right heart failure. Serological tests for hepatitis A, B and C were negative as were tests for herpes simplex virus, Epstein Barr virus and cytomegalovirus. The same was true for the autoantibody panel (antinuclear antibodies, antineutrophil cytoplasmatic antibodies, liverkidney membrane antibodies, soluble liver antigen antibodies and antimitochondrial antibodies were all negative).

As shown in figure 1, the first laboratory tests 2 weeks after discontinuation of sitaxentan revealed a slight decline in the ALT and AST levels. However, 2 weeks later both enzyme levels markedly increased and after a further 2 weeks the ALT levels had risen to almost $1,000 \mathrm{U} \cdot \mathrm{L}^{-1}$. At that time, bilirubin levels rose from normal $\left(2-14 \mu \mathrm{mol} \cdot \mathrm{L}^{-1}\right)$ to $30 \mu \mathrm{mol} \cdot \mathrm{L}^{-1}$ and the patient reported mild abdominal discomfort. The decision was made to start oral prednisolone therapy at a dose of $50 \mathrm{mg} \cdot \mathrm{day}^{-1}\left(\sim 1 \mathrm{mg} \cdot \mathrm{kg}^{-1}\right)$. Within 1 week, the aminotransferase levels had declined substantially (fig. 1). Prednisolone was tapered over a 4-week period and aminotransferase levels, as well as bilirubin levels, completely normalised and remained in the normal range thereafter.

In the present case, it is unlikely that hepatotoxicity was caused by a simple toxic mechanism as liver injury worsened for several weeks after drug discontinuation. The time course of liver injury and the response to corticosteroid therapy were suggestive of an immune-mediated mechanism. Obviously, it is impossible to verify whether or not this hypothesis is true, especially since a liver biopsy was not obtained. It is also

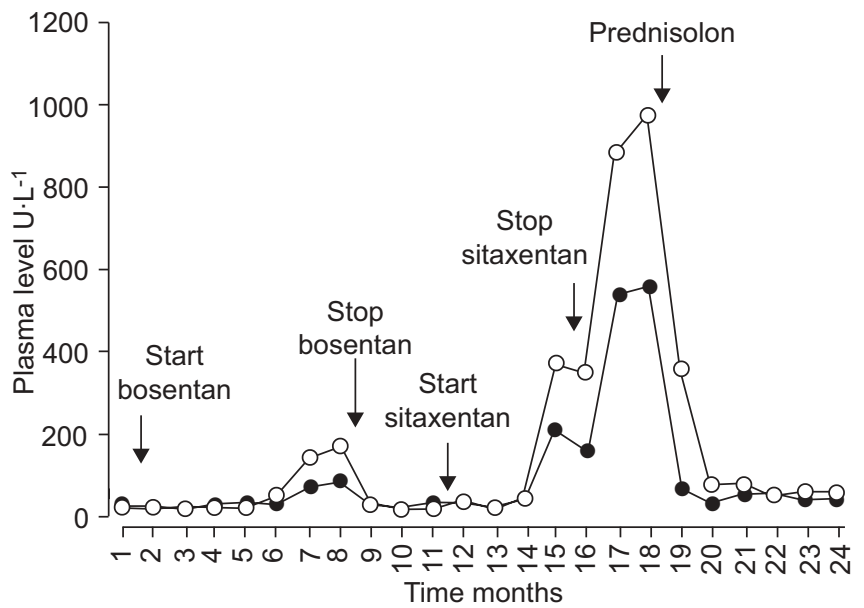

FIGURE 1. Course of hepatic aminotransferases. : aspartate aminotransferase; $\bigcirc$; alanine aminotransferase. 
unclear whether the sequential therapy with bosentan and sitaxentan was related to the severity of liver injury in this case, although previous case studies suggest that it is safe to administer sitaxentan in patients with elevated liver enzymes during bosentan therapy [6].

The present case report should not encourage physicians to treat endothelin receptor antagonists-related liver injury with steroids on a routine basis since the vast majority of these cases need no intervention other than dose reduction or drug discontinuation. However, the present case raises the possibility that idiosyncratic mechanisms might sometimes be involved in endothelin receptor antagonists-associated liver injury.

M.M. Hoeper*, K.M. Olsson*, A. Schneider ${ }^{*}$ and H. Golpon* *Depts of Respiratory Medicine, and "Gastroenterology, Hepatology and Endocrinology, Hannover Medical School, Hannover, Germany.

\section{STATEMENT OF INTEREST}

Statements of interest for all authors of this manuscript can be found at www.erj.ersjournals.com/misc/statements.dtl

\section{REFERENCES}

1 Dupuis J, Hoeper MM. Endothelin receptor antagonists in pulmonary arterial hypertension. Eur Respir J 2008; 31: 407-414.

2 Wilkins MR, Wharton J, Grimminger $\mathrm{F}$, et al. Phosphodiesterase inhibitors for the treatment of pulmonary hypertension. Eur Respir J 2008; 32: 198-209.

3 Olschewski H, Gomberg-Maitland M. Prostacyclin therapies for the treatment of pulmonary arterial hypertension. Eur Respir J 2008; 31: 801-901.

4 Humbert M, Segal ES, Kiely DG, et al. Results of European post-marketing surveillance of bosentan in pulmonary hypertension. Eur Respir J 2007; 30: 338-344.

5 Fattinger K, Funk C, Pantze M, et al. The endothelin antagonist bosentan inhibits the canalicular bile salt export pump: a potential mechanism for hepatic adverse reactions. Clin Pharmacol Ther 2001; 69: 223-231.

6 Benza RL, Mehta S, Keogh A, et al. Sitaxsentan treatment for patients with pulmonary arterial hypertension discontinuing bosentan. J Heart Lung Transplant 2007; 26: 63-69.

DOI: 10.1183/09031936.00193308 